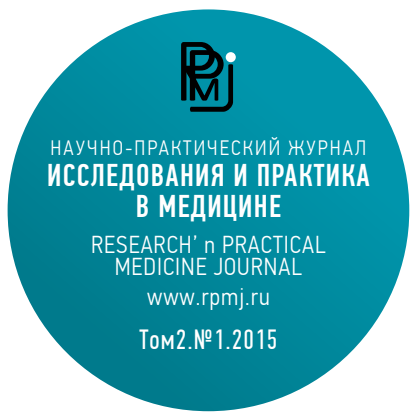

Ключевые слова:

Хромогранин А,

нейроэндокринная

дифференцировка,

рак предстательной железы

Keywords:

Chromogranin A,

neuroendocrine differentiation, prostate cancer

DOI:10.17709/2409-2231-2015-2-1-55-60

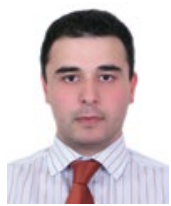

Для корреспонденции:

Кешишев Николай Георгиевич -

к.м.н., заведующий инновационным отделом НИИ урологии и интервенционной радиологии им. Н.А. Лопаткина - филиал ФГБУ «НМИРЦ» Минздрава России

Адрес: 105425, Российская Федерация,

г. Москва, ул. 3-я Парковая, д. 51, стр. 4.

Телефон: +7 4991649613

E-mail: nkeshishev@gmail.com

Статья поступила 07.02.2015,

принята к печати 05.03.2015

For correspondence:

Nikolay Keshishev -

$\mathrm{PhD}$, chief of innovative department

N. Lopatkin SRIUIR

Address: 51-4, 3-ya Parkovaya, Moscow,

Russian Federation, 105425

E-mail: nkeshishev@gmail.com

\section{ХРОМОГРАНИН-А В ДИАГНОСТИКЕ РАКА ПРЕДСТАТЕЛЬНОЙ ЖЕЛЕЗЫ}

\author{
Сивков А.В., Кешишев Н.Г., Ефремов Г.Д., Ковченко Г.А., Трудов А.А.,
} Никонова Л.М., Ромих Ф.Д.

НИИ урологии и интервенционной радиологии им. Н.А. Лопаткина - филиал ФГБУ «НМИРЦ» Минздрава России (Москва, Российская Федерация)

105425, Российская Федерация, г. Москва, ул. 3-я Парковая, д. 51, стр. 4

\section{Резюме:}

Основным маркером нейроэндокринной дифференцировки (НЭД) является хромогранин-А (ХгА).

Целью настоящей работы является определение роли НЭД при заболеваниях предстательной железы по уровню ХгА.

Материалы и методы. В исследование было включено 304 мужчины с различными заболеваниями ПЖ: ДГПЖ - 20; хронический простатит (ХП) - 22; ДГПж и простатическая интраэпителиальная неоплазия низкой степени (ДГПж+ПИННС) - 50; ДГПж и ПИН высокой степени (ДГПж+ПИНВС) 32; локализованный РПЖ (ЛРПЖ) - 80; местнораспространенный РПЖ (МРРПЖ) - 21; КРРПЖ - 51. Последняя группа объединяла в себе больных ЛРПЖ, МРРПЖ и метастатическим РПж. Всем пациентам указанных групп осуществляли забор крови натощак с дальнейшим определением уровня ХгА в сыворотке методом иммуноферментного анализа. Референсные значения ХгА для данного метода составляют от 0 до 3 нмоль/л.

Основные результаты. При анализе результатов исследования выявлена общая тенденция увеличения средних значений ХгА сыворотки крови по мере нарастания стадии РПЖ, при максимальном значении у пациентов с КРРПЖ. По результатам анализа не выявлено достоверной корреляции между возрастом и ХгА $(r=0,05)$ среди всех исследуемых групп. Отмечается стойкая корреляция $(r=0,32 ; p<0,001)$ между ХгА и уровнем ПСА среди всех изучаемых групп пациентов. Прослеживается некоторая зависимости между суммой баллов по шкале Глисона и ХгA $(r=0,19 ; p<0,05)$ при ЛРПЖ, МРРПЖ и КРРПЖ. Определяя зависимость между ХгА и стадией РПЖ (ЛРПЖ, МРРПЖ, КРРПЖ), мы также выявили корреляцию $(r=0,31, p<$ $0,005)$ среди этих групп пациентов.

Заключение. ХгА представляет высокий интерес для раннего выявления КРРПЖ. Также при помощи ХгА достаточно перспективно обнаружение наиболее агрессивных форм РПЖ. Определение НЭД опухоли может стать необходимым анализом для комплексного подхода к выбору тактики лечения РПЖ.

\section{CHROMOGRANIN-A IN THE DIAGNOSIS OF PROSTATE CANCER}

Morgunov L.Ju.Sivkov A. V., Keshishev N.G., Efremov G.D., Kovchenko G.A., Trudov A. A., Nikonova L. M., Romikh F. D.

N. Lopatkin Scientific Research Institute of Urology and Interventional Radiology - branch of the National Medical Research Radiological Centre of the Ministry of Health of the Russian Federation (Moscow, Russian Federation) 51, str 4, ul. 3-ja Parkovaja, Moscow, Russian Federation, 105425

\section{Summary}

The principal marker of neuroendocrine differentiation (NED) is chromogranin A (HGA). The purpose of this work is to determine the role of NED in diseases of the prostate gland by the level of HGA.

Materials and methods. The study included 304 men with various diseases of the prostate: $\mathrm{BPH}-20$; chronic prostatitis (CP) - 22; BPH and prostatic lowgrade intraepithelial neoplasia (BPH+LDPIN) - 50; $\mathrm{BPH}$ and high degree PIN (BPH+HGPIN) - 32; localized prostate cancer (LPC) - 80; locally advanced PCA 
(LAPC) - 21; CRPC - 51. The last group United in patients LPC, LAPC and metastatic prostate cancer. All these groups of patients were performed blood sampling fasting with further definition of the level of HGA in serum by enzyme immunoassay. The reference values of HGA for this method are ranged from 0 to $3 \mathrm{nmol} / \mathrm{L}$.

Results. During the analyzing of the results of the study they revealed a general trend of increasing of mean values of HGA of serum with the growth stage of prostate cancer, with a maximum of patients with CRPC. According to the analysis they revealed no significant correlation between age and HGA $(r=0,05)$ among all study groups. They noted the persistent correlation $(r=0,32 ; p<0,001)$ between
HGA and PSA levels among all studied groups of patients. They traced some relationship between the amount of points Gleason and HGA $(r=0,19 ; p<0,05)$ in cases of LPC, LAPC and CRPC. Defining the relationship between HGA and stage of prostate cancer (LPC, LAPC and CRPC), we also found a correlation $(r=0,31, p<0,005)$ among these groups of patients.

Conclusions. HGA is a high interest for the early detection CRPC. Also with the help of HGA it seem to be quite promising to discover the most aggressive forms of prostate cancer. Definition of NED-tumor may be necessary for the analysis of a comprehensive approach to prostate cancer treatment selection.
Первое упоминание о нейроэндокринных клетках (НЭК) сделано Pretl К. в 1944 г. (Pretl, 1944). С тех пор данные клетки были описаны в легких, пищеварительном тракте, поджелудочной железе, предстательной железе и других органах. В норме нейроэндокринные клетки присутствуют во всех отделах предстательной железы (Пж) при рождении. Располагаясь рассеянно в ткани железы, число НЭК значительно увеличивается во время пубертатного периода, после чего остается относительно неизменным в возрастном интервале 25-54 года (Sciarra, et al., 2011). НЭК встречаются во всех отделах предстательной железы (ПЖ), однако прослеживается общая тенденция к их большему скоплению в области крупных протоков железы, и меньшему - в ацинарной ткани. Таким образом, отмечается некая неравномерность распределения НЭК, в том числе и в здоровой ткани ПЖ (Battaglia, et al., 1994).

Согласно морфологическому представлению существует два типа НЭК ПЖ: клетки «открытого» типа колбообразной формы, достигающие просвета протока, а также клетки «закрытого» типа, не достигающие просвета. Клетки обоих типов имеют сложное строение и сообщаются с соседними эпителиальными клетками при помощи дендритоподобных отростков (Abrahamsson, 1999;).

В 1999 году авторами Aumuller G. Et al. было показано, что НЭК имеют нейрогенное происхождение, однако, мнения исследователей расходятся и единой позиции по этому вопросу пока нет (Aumuller, et al., 1999).

Основными маркерами НЭК являются: хромогранин-А (ХгА), серотонин, секретогранин, бомбезин, тиреоидстимулирующий гормон-подобный пептид, кальцитонин, катакальцин, паратиреоидный гормон-связанный пептид и др. Среди данных субстанций наиболее ценным и изученным маркером выявления нейроэндокринной дифференцировки (НЭД) рака предстательной железы (РПЖ) является ХгА (Sciarra, et al., 2011; Hansson, Abrahamsson, 2001).

Хромогранины относятся к семейству гликопротеинов и являются представителями гранинов - кислых секреторных белков. Различают следующие типы хромогранинов: хромогранин А (ХГА); хромогранин В (ХгВ или секретогранин I), хромогранин С (ХГC или секретогранин II). Они обнаруживаются в везикулах и высвобождаются при экзоцитозе, способствуя структурированию пептидов, гормонов и нейропептидов (Eriksson, et al., 1989).
Наибольший клинический интерес вызывают ХгА и ХгВ. Хромогранины встречаются во всех эндокринных клетках, особенно в нейронах центральной и периферической нервной системы. В равной степени экспрессируются ХгА и ХгВ в хромаффинных клетках надпочечников. В клетках паращитовидной железы и в нейросекреторных клетках желудка ХгА содержится в большем количестве, чем ХгВ.

ХгА - гликопротеин, состоящий из 439 аминокислотных остатков. По всей длине молекулы распределены основные аминокислотные остатки, особенно ими богат С-конец молекулы. По этим аминокислотным остаткам происходит расщепление молекулы, в результате чего образуются вазостатин и хромостатин. ХгВ сходен по химической структуре с ХГА, но распространен менее широко. При нейроэндокринных опухолях, как правило, отмечают увеличение в сыворотке крови ХгА, а иногда и ХгВ (Abrahamsson, 1999b; Angelsen, et al., 1997).

Наиболее информативным иммунологическим анализом при диагностике опухоли является измерение уровня ХгА, уровень которого в сыворотке крови повышается вне зависимости от типа опухоли, секретирующей или не секретирующей (Di Sant'Agnese, 1995). Иммуногистохимический метод позволяет визуализировать ХгА в клетках опухоли, в том числе при раке предстательной железы (РПЖ).

В 2011 году были опубликованы данные, согласно которым уровень ХгА в крови может изменяться под влиянием различных факторов или патологических состояний, не связанных с предстательной железой. ХгА может повышаться при: гастро-энтеро-панкреатических опухолях, нейроэндокринных опухолях поджелудочной железы, синдроме множественной эндокринной неоплазии 1 типа, опухолях мозгового слоя надпочечников, опухоли паращитовидных желез, а также у больных co сниженной функцией почек (Peracchi, et.al., 2003).

Среди факторов, вызывающих существенное увеличение концентрации ХгА крови, можно выделить: лечение ингибиторами протонной помпы или блокаторами Н2-рецепторов, хронический атрофический гастрит (тип A), нарушение функции почек, ревматоидный артрит с высоким уровнем ревматоидного фактора и IgМ. Умеренное или слабое влияние на повышение уровня ХгА оказывают воспалительные заболевания кишечника (язвенный колит и болезнь Крона), нарушение функции печени, не леченная гипертоническая болезнь, сердеч- 
ная недостаточность, гиперкортицизм и, по некоторым данным, процесс переваривания пищи в кишечнике (Glinicki, Jeske, 2010). Кроме того, характер биологического материала (сыворотка или плазма) также имеет большое значение. Ценность ХгА в диагностике РПж изучают на протяжении многих лет. По этому вопросу накоплены определенные знания, хотя и неоднозначные по некоторым аспектам.

Kadmon D. et al. представили работу, в которой указали на то, что ХгА - это основной нейроэндокринный белок, в 48\% продуцируемый метастатическими опухолями (Kadmon, et al., 1991). Позже были опубликованы работы, где определение данного маркера в крови больного РПЖ указывает на НЭД не только метастатической опухоли, но и первичного РПЖ (Ischia, et al., 2000). Некоторые авторы утверждают, что ХгА - это независимый прогностический маркер в клиническом стадировании РПЖ (Ferrero-Pous, et al., 2001), в то время как другие не согласны с данным утверждением (Sciarra, et al., 2004). Повышение в сыворотке крови ХгА чаще встречается у пациентов с кастрационно-рефрактерным раком предстательной железы (КРРПЖ). Кроме того, существует достоверная корреляция между экспрессией нейроэндокринных маркеров и отдаленными метастазами (Cussenot, et al., 1996). Пациенты с РПЖ с высоким уровнем ХгА имеют достоверно худший прогноз, по сравнению с теми больными, у которых НЭД опухоли не выявляется (Cussenot, et al., 1996).

Angelsen A. et al. выявили корреляцию между количеством данного маркера в НЭК и сывороточным ХгА у мужчин с РПЖ, где повышение тканевого ХгА, соответственно, сопровождалось повышением сывороточного (Angelsen, et al., 1997)

В работе Appetecchia M. et al., в которую авторы включили данные 486 пациентов, было проведено исследование, демонстрирующее корреляцию хромогранина А, ПСА и сумму баллов по шкале Глисона в сыворотке крови у пациентов с неметастатическим РПЖ. Авторы отметили, что уровень сывороточного ХгА достоверно не коррелировал с ПСА $(p=0,44)$ и рТ-стадией $(p=0,89)$, однако его увеличение наблюдалось при росте суммы баллов по шкале Глисона: при Gl<7 увеличение уровня ХгА отмечено 25,5\% больных, при GI >7 - у 31,4\%. Таким образом, уровень этого маркера коррелировал с ростом суммы баллов и, возможно, с агрессивностью РПЖ. Определение ХгА перед выбором лечения может существенно дополнять диагностические данные в прогнозировании более агрессивного течения заболевания (Appetecchia, et al., 2010).

Tarjan M. провел исследование, в которое вошло 40 пациентов в равных пропорциях с метастатическим и неметастатическим РПЖ. Во всех 40 случаях проводился иммуногистохимический анализ ХгА при пересмотре гистологического материала. Его повышение было выявлено в 14 из 20 случаев РПЖ с метастазами (78\%). Интересен факт, что при неметастатическом РПЖ экспрессии хромогранина А ни в одном случае выявлено не было. По итогам работы было сделано заключение, что НЭД опухоли ПЖ, очевидно, является признаком агрессивного и неблагоприятного течения заболевания (Tarjan, 2010).
В исследовании Wu J. et al. осуществлялся мониторинг динамики роста уровня маркера в крови 14 пациентов с КРРПЖ. Повышение уровня ХгА на ранних этапах гормонального лечения наблюдалось у 10 пациентов (71\%), в то время как уровень общего ПСА оставался ниже 10 нг/мл. Таким образом, данный хромогранин можно отнести к раннему маркеру КРРПЖ (Wu, et al., 1998).

В другом исследовании, проводимом Chuang C. et al. с октября 1998 года по январь 2003 года, участие принимали 90 пациентов с локализованным ( $\mathrm{n}=20$ ) и метастатическим ( $\mathrm{n}=70$ ) РПЖ. Все пациенты получали андроген-депривационную терапию. Уровень ПСА и ХгА определялся перед лечением и каждые три месяца во время проводимого гормонального лечения. Средний период наблюдения составил 35 месяцев. У 36 пациентов $(46,2 \%)$ во время лечения уровень ПСА не поднимался выше 4 нг/мл, а уровень ХгА составил менее 84,6 нг/мл при норме до 100 нг/мл в данной лаборатории. У 17 пациентов (21,8\%) уровень ПСА также не поднимался, однако уровень ХгА прогрессивно увеличивался. У остальных 25 человек (32\%) развилась резистентность к проводимой гормональной терапии, у 17 мужчин наблюдался прогрессивный подъем уровня ХгА (более 100 нг/мл), предшествовавший подъему ПСА в среднем на десять месяцев. Следовательно, ХгА может на ранних этапах указывать на неэффективность гормональной терапии, в то время как уровень ПСА сохраняется на низком уровне (Chuang, et al., 2003).

Cabrespine A. et al. для определения НЭД опухоли у 39 пациентов оценивали уровень данного маркера в динамике до и во время проводимого гормонального лечения. Повышение уровня ХгА было выявлено в $45 \%$ случаев, хотя достоверной корреляции между его уровнем и уровнем ПСА не обнаружено. При этом корреляция между повышенным хромогранином А и снижением эффективности в ответ на гормональную терапию наблюдалась в 25\% случаев. По итогам работы авторы пришли к заключению, что этот маркер позволяет на ранних этапах заподозрить КРРПЖ (Cabrespine, et al., 2006). Bocan E. et al. в своем труде указывают на то, что с ростом суммы баллов по шкале Глисона повышается и уровень ХгA. (Bocan, et al., 2011). При десятилетнем наблюдении за 140 пациентами, которым была выполнена радикальная простатэктомия с лимфодиссекцией и в последующем иммуногистохимический анализ ХгA, Quek M.L. et al. пришли к выводу, что предоперационный ПСА и патогистологическое стадирование достоверно не коррелировали с десятилетней выживаемостью пациентов, в то время как НЭД опухоли наряду с наличием метастазов в лимфатических узлах достоверно сопровождалась более низкой выживаемостью (Quek, et al., 2006).

Также крупное исследование было проведено Alessandro S., где автор указал на то, что определение предоперационного сывороточного ХгА может выступать в качестве независимого прогностического маркера раннего биохимического рецидивирования РПЖ после радикальной простатэктомии. В этом исследовании принимали участие 264 пациента с неметастатическим РПж, которым выполнялась радикальная простатэктомия. До операции всем больным определялся уровень ПСА 
и ХгА. В послеоперационном периоде биохимическая прогрессия отмечалась у 19,4\% пациентов с уровнем ХгА $\leq 90 \mathrm{Hг} / \mathrm{Mл}$, а у пациентов с уровнем >90 нг/мл прогрессия наблюдалась в 64,7\% случаев (Alessandro, et al., 2007).70

В другом исследовании проводилось сравнение уровня сывороточного ХгА у 57 пациентов с верифицированным РПЖ в различных стадиях опухолевого процесса и у 22 пациентов без РПЖ. Его медиана была достоверно выше у пациентов с РПЖ ( $p=0,0271)$, а уровень ХгА коррелировал со стадией РПЖ (Sugimoto, et al., 2007).

\section{Цель исследования.}

В НИИ урологии нами проведено эпидемиологическое исследование, целью которого стало определение средних значений показателя ХгА сыворотки крови при различных заболеваниях предстательной железы. В доступной научной литературе такой информации найдено не было.

\section{Материалы и методы}

В исследование включено 304 мужчины с различными заболеваниями ПЖ: ДГПЖ - 20; хронический простатит (ХП) - 22; ДГПЖ и простатическая интраэпителиальная неоплазия низкой степени (ДГПЖ+ПИННС) 50; ДГПЖ и ПИН высокой степени (ДГПЖ+ПИНВС) - 32; локализованный РПЖ (ЛРПЖ) - 80; местнораспространенный РПЖ (МРРПЖ) - 21; КРРПЖ - 51. Последняя группа объединяла в себе больных ЛРПЖ, МРРПж и метастатическим РПЖ. Пациентам всех вышеуказанных групп диагноз был установлен клинически и подтвержден при патоморфологическом исследовании. Отдельную контрольную группу составили 28 здоровых мужчин - добровольцев. Всем пациентам указанных групп осуществляли забор крови натощак с дальнейшим определением уровня ХгА в сыворотке методом иммуноферментного анализа с использованием наборов «Human Chromogranin-A ELISA Kit» (Eurodiagnostica, Швеция) на анализаторе "Gemini» (Stratec Biomedical Systems, Швейцария). Референсные значения ХгА для данного метода составляют от 0 до 3 нмоль/л. Также всем больным были выполнены клинический и биохимический анализы крови для выявления сопутствующих патологических процессов, а также определен уровень простат-специфического антигена (ПСА) сыворотки крови. С учетом анамнеза у пациентов исключали заболевания желудочно-кишечного тракта, эндокринной системы, онкологические процессы, неконтролируемую артериальную гипертензию, предшествующие хирургические манипуляции на органах мочевой системы. При обнаружении указанных патологий пациенты не включались в исследование.

\section{Результаты}

При анализе результатов исследования выявлена общая тенденция увеличения средних значений ХгА сыворотки крови по мере нарастания стадии РПЖ, при максимальном значении у пациентов с КРРПЖ (табл. 1).

Не получено достоверных различий в уровнях ХгА крови между мужчинами контрольной группы, пациентами с ХП и ДГПж, а также с ДГпЖ+ПИННС и ДГПж+ПИНВС:

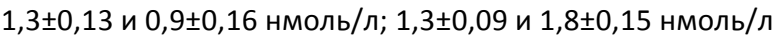

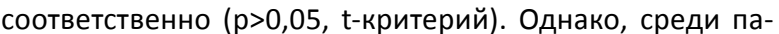
циентов с ДГПЖ+ПИНВС, число имевших уровень ХгА более 3 нмоль/л оказалось выше, чем среди страдающих ДГПЖ+ПИННС: 9,3 и 4\%, соответственно ( $p=0,05 *)$. Сравнительный анализ среднего уровня ХгА у пациентов с ЛРПЖ и МРРПЖ показал наличие достоверной разницы: 1,8 и 2,7 нмоль/л соответственно ( $<<0,05$, t-критерий). При КРРПЖ средний уровень ХгА сыворотки крови был выше, чем при МРРПЖ и ЛРПЖ: 3,4 нмоль/л; 2,7 нмоль/л и 1,8 нмоль/л соответственно ( $<<0,05$ и p $<0,05$, t-критерий). Таблица 1 наглядно демонстрирует значения и достоверность различий средних показатели ХгА сыворотки крови в сравнении с ПСА при различных заболеваниях Пж.

Число пациентов, демонстрирующих уровень ХгА более 3 нмоль/л, при КРРПж также оказалось выше, чем среди пациентов с МРРПЖ и ЛРПЖ, составило 34, 29 и 11,5\% соответственно и было статистически достоверно $(p<0,05 ; p<0,01)$ по ТМФ-критерию (рис. 1).

Таблица 1

Средние показатели ПСА и ХгА сыворотки крови в зависимости от состояния ПЖ.

\begin{tabular}{|c|c|c|c|c|c|}
\hline Диагноз & $\begin{array}{c}\text { Число } \\
\text { пациентов }\end{array}$ & ПСА (нг/мл) & $\begin{array}{c}\text { ХгА (нмоль/л) (референсное } \\
\text { значение - до } 3 \text { нмоль/л) }\end{array}$ & \multicolumn{2}{|c|}{ Достоверность различий } \\
\hline Контрольная группа & 28 & 0,7 & $1,3 \pm 0,11$ & & \\
\hline дгпж & 20 & 5,1 & $0,9 \pm 0,16$ & \multirow[t]{2}{*}{$p>0,05^{*}$} & \multirow[t]{4}{*}{$p>0,05^{*}$} \\
\hline Хронический простатит & 22 & 5,7 & $1,3 \pm 0,13$ & & \\
\hline ДГпж + ПиннС & 50 & 6,8 & $1,3 \pm 0,09$ & \multirow[t]{2}{*}{$p>0,05^{*}$} & \\
\hline ДГПж + ПИнВС & 32 & 11,8 & $1,8 \pm 0,15$ & & \\
\hline Локализованный РПЖ & 80 & 9,8 & $1,8 \pm 0,14$ & \multirow[t]{2}{*}{$p>0,05^{*}$} & \multirow[t]{3}{*}{$p>0,05^{*}$} \\
\hline Местнораспространенный РПЖ & 21 & 12,0 & $2,7 \pm 0,53$ & & \\
\hline КРРПЖ & 51 & 192,9 & $3,4 \pm 0,42$ & & \\
\hline Общее число пациентов & 304 & & & & \\
\hline
\end{tabular}

*достоверность различий по U критерию Вилкоксона-Манна-Уитни 
Полученные статистические результаты требуют дальнейшего уточнения, так как в их основе лежит предположение о нормальном распределении данных. В связи с этим, при помощи тестов, основанных на характеристиках распределения, была проведена проверка гипотезы о нормальном распределении анализируемых показателей (HN). Использовали критерии асимметрии и эксцесса распределения. (Kotz, et al., 2006). Нулевая гипотеза с уровнем значимости в 5\% была отвергнута во всех случаях, кроме группы ДГПЖ+ПИНВС на основе критерия эксцесса. Полученные результаты позволяют сделать следующие выводы:

- нет предпосылок принять гипотезу о нормальном распределении данных;

- для подтверждения преимущественно монотонного роста средних значений исследуемого маркера желательно привлечение непараметрических статистических процедур, не опирающихся на предположение о том, что данные имеют нормальное распределение.

С целью выявления статистической связи параметров возраста, ПСА, ХгА сыворотки крови при всех исследуемых заболеваниях ПЖ, а также показателя Глисона при РПж, был проведен визуальный анализ соответствующих регрессионных зависимостей. Для формального обоснования наличия взаимосвязей использовался непараметрический критерий значимости, основанный на статистике Спирмена.

Для удобства обработки данных и отображения графических результатов мы шифровали имеющиеся группы пациентов в виде целых цифровых переменных от 0 до 7, где 0 - контрольная группа, 1 - ДГпж, 2 - Хронический простатит, 3 - ДГПЖ+ПИННС, $4-$ ДГПЖ+ПИНВС, 5 - ЛРПЖ, 6 - МРПЖ, 7 - КРРПЖ

По результатам анализа не выявлено достоверной корреляции между возрастом и ХгA $(r=0,05)$ среди всех исследуемых групп. Однако отмечается стойкая корреляция $(r=0,32 ; p<0,001)$ между ХгА и уровнем ПСА среди всех изучаемых групп пациентов. Также прослеживается некоторая зависимости между суммой баллов по шкале Глисона и ХгА $(r=0,19 ; \mathrm{p}<0,05)$ при ЛРПЖ, МРРПЖ и КРРПЖ. Определяя зависимость между ХгА и стадией РПЖ (ЛРПЖ, МРРПЖ, КРРПЖ), мы также выявили корреляцию $(r=0,31, p<0,005)$ среди этих групп пациентов.

\section{Обсуждение}

Существует множество публикаций, посвященных изучению НЭК РПЖ, а также поиску оптимального нетканевого маркера НЭД. Во многих работах показано, что ХгА не отражал наличие РПж и коррелировал только с возрастом пациентов. В то же время, существует большое число исследований, которые утверждают, что ХгА коррелирует и со стадией и со степенью агрессивности РПЖ.

Нам впервые удалось получить убедительные данные средних значений уровней ХгА сыворотки крови при различных заболеваниях Пж и выявить достоверную связь увеличения показателей ХгА с развитием злокачественного процесса в органе. Мы показали, что достоверное увеличение среднего уровня ХгА крови происходит по мере прогрессии РПЖ: от локализованных форм - до КРРПЖ. При этом не было выявлено статистически значимых отличий показателей ХгА при ДГПЖ, ХП, ДГПЖ+ПИННС, ДГПЖ+ПИНВС и контрольной группой. В ходе статистической обработки полученных данных установлена корреляция ХгА с уровнем ПСА, суммой баллов по шкале Глисона и стадией РПЖ.

Настоящее исследование демонстрирует, что ХгА является важным маркером в диагностике РПж и достоверно соотносится с распространенностью опухолевого процесса. Наибольшее повышение ХгА крови наблюдали при наступлении резистентности к гормональной терапии, что, очевидно, обусловлено ростом пула НЭК в опухолевой ткани. Основываясь на данных проведенного исследования считаем оправданным определение уровня ХгА сыворотки крови на начальном этапе диагностики РПЖ.

\section{Заключение}

Таким образом, из представленного обзора становится ясно, что повышение Хромогранина А имеет высокую ценность для определения НЭД РПЖ, особенно при КРРПЖ. Также, при помощи ХгА достаточно перспективно обнаружение наиболее агрессивных форм РПЖ. Наряду с такими стандартными тестами, как уровень ПСА, сумма баллов по шкале Глисона, количество позитивных столбиков, определение НЭД опухоли может стать необходимым маркером комплексного подхода к лечению РПж.

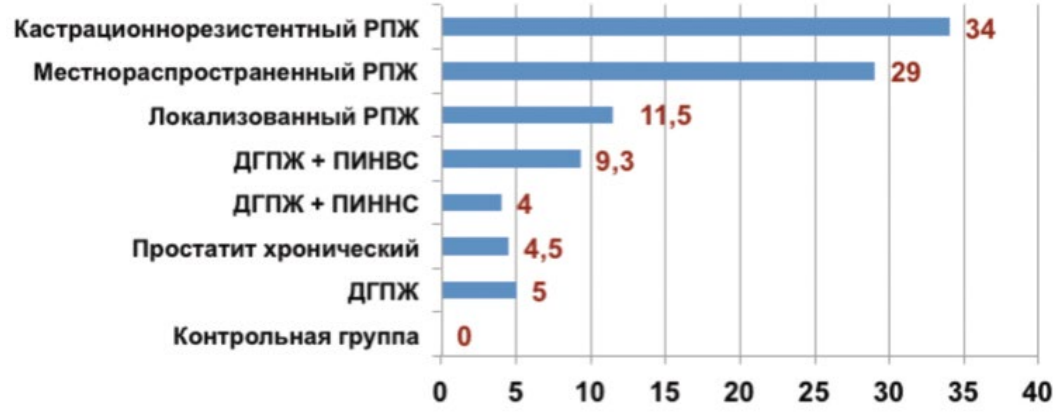

Рисунок 1. Доля больных (в \%) с повышенным уровнем ХГА при различных заболеваниях ПЖ 
Список литературы/References:

1. Abrahamsson P. A. Neuroendocrine cells in tumour growth of the prostate//Endocr Relat Cancer. 1999a. Vol. 6. P. 503-519.

2. Abrahamsson P. A. Neuroendocrine differentiation in prostatic carcinoma//Prostate. 1999b. Vol. 39. P. 135-148.

3. Alessandro S., Vincenzo G., Maria A. G., et al. Chromogranin A and biochemical progressionfree survival in prostate adenocarcinomas submitted to radical prostatectomy.//Endocr Relat Cancer. 2007. Sep. Vol. 14. №. 3. P. 625-632.

4. Angelsen A., Syversen U., Haugen O.A., et al. Neuroendocrine differentiation in carcinomas of the prostate: do neuroendocrine serum markers reflect immunohistochemical findings?//Prostate. 1997. Vol. 30. №. 1. P. 1-6.

5. Angelsen A., Syversen U., Stridsberg M., et al. Use of neuroendocrine serum markers in the follow-up of patients with cancer of the prostate.//Prostate. 1997. Vol. 31. №. 2. P. 110-117.

6. Appetecchia M., Mecule A., Pasimeni G., et al. Incidence of high chromogranin A serum levels in patients with non metastatic prostate adenocarcinoma//J Exp Clin Cancer Res. 2010. Vol. 29. P. 166.

7. Battaglia S., Casali A.M., Botticelli A.R. Age-related distribution of endocrine cells in the human prostate: a quantitative study//Virchows Arch. 1994. Vol. 424. №. 2. P. 165-168.

8. Bocan E. V., Mederle O., Sarb S., et al. Correlation between histopathological form and the degree of neuroendocrine differentiations in prostate cancer.//Rom J Morphol Embryol. 2011. Vol. 52. №. 4. P. 1215-1218.

9. Bonkhoff H., Stein U., Remberger K. Multidirectional differentiation in the normal, hyperplastic, and neoplastic human prostate: simultaneous demonstration of cell-specific epithelial markers//Hum Pathol. 1994 Jan. Vol. 25. №. 1. P. 42-46.

10. Cabrespine A., Guy L., Gachon F., et al. Circulating chromogranin a and hormone refractory prostate cancer chemotherapy.//J Urol. 2006. Vol. 175. №. 4. P. 1347-1352.

11. Cabrespine A., Guy L., Gachon F., et al. Circulating chromogranin a and hormone refractory prostate cancer chemotherapy.//J Urol. 2006. Vol. 175. №. 4. P. 1347-1352.

12. Chuang C. K., Wu T. L., Tsao K. C. Elevated serum chromogranin A precedes prostate-specific antigen elevation and predicts failure of androgen deprivation therapy in patients with advanced prostate cancer.//J Formos Med Assoc. 2003. Vol. 102, №. 7. P. 480-485.

13. Denis L., Griffiths K., Khoury S., et al. Benign Prostatic Hyperplasia (BPH)//Paris. 1997. P. 95-96.

14. Di Sant'Agnese P. Neuroendocrine differentiation in prostatic carcinoma//Recent findings and new concepts. Cancer 1995. Vol. 75. P. 1850-1959.

15. Eriksson B., Arnberg H., Oberg K., et al. Chromogranins - new sensitive markers for neuroendocrine tumors//Acta Oncol. 1989. Vol. 28. №. 3. P. 325-329.

\section{Информация об авторах:}

1. Сивков Андрей Владимирович - к.м.н., первый заместитель директора по науке НИИ урологии и интервенционной радиологии им. Н. А. Лопаткина - филиал ФГБУ «НМИРЦ» Минздрава России

2. Кешишев Николай Георгиевич - к.м.н. заведующий инновационным отделом НИИ урологии и интервенционной радиологии им. Н. А. Лопаткина - филиал ФГБУ «НМИРЦ» Минздрава России

3. Ефремов Геннадий Дмитриевич - к.м.н., заведующий патоморфологической лаборатории НИИ урологии и интервенционной радиологии им. Н.А. Лопаткина - филиал ФГБУ «НМИРЦ» Минздрава России

4. Ковченко Григорий Александрович - м. н.с. инновационного отдела НИИ урологии и интервенционной радиологии им. Н. А. Лопаткина - филиал ФГБУ «НМИРЦ» Минздрава России

5. Трудов Алексей Александрович - м.н.с. инновационного отдела НИИ урологии и интервенционной радиологии им. Н.А. Лопаткина - филиал ФГБУ «НМИРЦ» Минздрава России

6. Никонова Лариса Марбековна - заведующая лабораторией
16. Ferrero-Pous M., Hersant A. M., Pecking A. Serum chromogranin - A in advanced prostate cancer.//BJU Int.. 2001. Vol. 88. №. 7. P. $790-796$.

17. Cussenot O., Villette J. M., Valeri A., et al. Plasma neuroendocrine markers in patients with benign prostatic hyperplasia and prostatic carcinoma//J Urol. 1996. Vol. 155. №. 4. P. 1340-1343.

18. Glinicki P., Jeske W. Chromogranin A (CgA) - the influence of various factors in vivo and in vitro, and existing disorders on it's concentration in blood//Endokrynol. Pol. 2010. Vol. 61. P. 384-387.

19. Hansson J., Abrahamsson P. A. Neuroendocrine pathogenesis in adenocarcinoma of the prostate//Ann Oncol. 2001. Vol. 12. Suppl 2. P. 145-152.

20. Ischia R., Hobisch A., Bauer R., et al. Elevated levels of serum secretoneurin in patients with therapy resistant carcinoma of prostate.//J Urol. 2000. Vol. 163. № 4. P. 1164-1165.

21. Kadmon D., Thompson T. C., Lynch G. R., Scardino P. T. Elevated plasma chromogranin - A concentrations in prostatic carcinoma.//J Urol. 1991. Vol. 146. № 2. P. 358-361.

22. Kotz S, Campbell B. Read, Balakrishnan N., Vidakovic B. (Eds.). Encyclopedia of statistical sciences. 16-volume set. 2nd Edition 2006. Vol.3. P. 1630-1631.

23. Peracchi M., Conte D., Gebbia C., et al. Plasma chromogranin A in patients with sporadic gastro-entero-pancreatic neuroendocrine tumors or multiple endocrine neoplasia type 1//Eur J Endocrinol. 2003. Vol. 148. №. 1. P. 39-43.

24. PretlK. Zur frage der endocrine menschlichen versteherdruse//Virchows Arch. 1944. Vol. 312. P. 392-404.

25. Quek M. L., Daneshmand S., Rodrigo S., et al. Prognostic significance of neuroendocrine expression in lymph node-positive prostate cancer.//Urology. 2006. Vol. 67. №. 6. P. 1247-1252.

26. Sciarra A., Innocenzi M., Ravaziol M., et al. Role of neuroendocrine cells in prostate cancer progression//Urologia. 2011. Vol. 78. №. 2. P. 126-131.

27. Sciarra A., Voria G., Monti S., et al. Clinical understaging in patients with prostate adenocarcinoma submitted to radical prostatectomy: predictive value of serum Chromogranin A//Prostate. 2004. Vol. 58. №. 4. P. 421-428.

28. Hirano D., Minei S., Sugimoto S., et al. Implications of circulating chromogranin A in prostate cancer. Hirano D., Minei S.,//Scand J Urol Nephrol. 2007. Vol. 41. №. 4. P. 297-301.

29. Tarjan M. Prognostic significance of focal neuroendocrine differentiation in prostate cancer: Cases with autopsy-verified cause of death//Indian J Urol. 2010. Vol. 26. №. 1. P. 41-45.

30. Wu J.T, Astill M. E., Liu G. H., Stephenson R. A. Serum chromogranin $A$ : early detection of hormonal resistance in prostate cancer patients.//J Clin Lab Anal. 1998. Vol. 12. №. 1. P. 20-25.

НИИ урологии и интервенционной радиологии им. Н.А. Лопаткина - филиал ФГБУ «НМИРЦ» Минздрава России

7. Ромих Филипп Дубровкович - лаборант инновационного отдела НИИ урологии и интервенционной радиологии им. Н. А. Лопаткина - филиал ФГБУ «НМИРЦ» Минздрава России

Information about co-authors:

1. Sivkov A. V. - Ph. D., Deputy Director for Science N. Lopatkin SRIUIR

2. Keshishev N.G.- Ph.D. Head of the division for innovative N. Lopatkin SRIUIR

3. EfremovG.D. - Ph.D., head of the Laboratory of Pathology N. Lopatkin SRIUIR

4. Kovchenko G.A.- Junior Researcher innovation Division N. Lopatkin SRIUIR

5. Trudov A. A. - Junior Researcher innovation Division N. Lopatkin SRIUIR

6. Nikonova L. M. - Head of the Laboratory N. Lopatkin SRIUIR

7. Romikh F. D. - Laboratory of Innovation N. Lopatkin SRIUIR 